\title{
Sistemas fuzzy complementam a deteç̧ão de socialbots por aprendizado de máquina
}

\section{Fuzzy Systems improve the accuracy of machine learning detection of socialbots}

\author{
Carla C. Pacheco ${ }^{1}$, Alex Garcia ${ }^{2 * *}$, Raphael Machado ${ }^{3}$ e Ronaldo M. Salles ${ }^{1 *}$ \\ ${ }^{1}$ Instituto Militar de Engenharia, Pós-Graduação em Engenharia de Defesa, Praça \\ General Tibúrcio, 80, Rio de Janeiro, RJ, Brasil. *orientador
}

${ }^{2}$ Instituto Militar de Engenharia, Seção de Engenharia da Computação, Praça General Tibúrcio, 80, Rio de Janeiro, RJ, Brasil. ** co-orientador

${ }^{3}$ Instituto Nacional de Metrologia, Qualidade e Tecnologia, Diretoria de Metrologia Científica e Tecnologia, Rua Santa Alexandrina, 416, Rio de Janeiro, RJ, Brasil

carlapacheco@globo.com, \{garcia, salles\}@ime.eb.br, rcmachado@inmetro.gov.br

\begin{abstract}
Machine learning has been widely used in the detection of socialbots in Online Social Networks. This paper presents the use of an algorithm committee to improve the accuracy of socialbots identification. The committee combines the knowledge obtained by machine learning algorithms and human heuristic knowledge obtained through interviews and formalized in fuzzy rules. Results show that these approaches are complementary, since their use in a single committee presents accuracy above 93\%, better than each of the algorithms independently.
\end{abstract}

Resumo. A deteç̧ão de socialbots em Redes Sociais Online tem sido objeto de diversos estudos baseados em aprendizado de máquina. Este trabalho apresenta o uso de um comitê de classificadores para melhorar a acurácia da identificação de socialbots. $O$ comitê associa o conhecimento obtido por algoritmos de aprendizado de máquina ao conhecimento heurístico humano, obtido por entrevistas e formalizado por regras fuzzy. Os resultados mostram que estas abordagens são complementares, uma vez que o uso conjunto destes algoritmos em um comitê apresenta uma acurácia acima de 93\%, maior do que os mesmos algoritmos utilizados isoladamente.

\section{Referência}

PACHECO, Carla de Castro; GARCIA, Alex de Vasconcellos; MACHADO, Raphael Carlos Santos; SALLES, Ronaldo Moreira. Sistemas fuzzy complementam a detecção de socialbots por aprendizado de máquina. Brasília: Revista Militar de Ciência e Tecnologia, 2018. 